\title{
SIMPLE HOPF ALGEBRAS AND DEFORMATIONS OF FINITE GROUPS
}

\author{
César Galindo and Sonia Natale
}

\begin{abstract}
We show that certain twisting deformations of a family of supersolvable groups are simple as Hopf algebras. These groups are direct products of two generalized dihedral groups. Examples of this construction arise in dimensions 60 and $p^{2} q^{2}$, for prime numbers $p, q$ with $q \mid p-1$. We also show that certain twisting deformation of the symmetric group is simple as a Hopf algebra. On the other hand, we prove that every twisting deformation of a nilpotent group is semisolvable. We conclude that the notions of simplicity and (semi)solvability of a semisimple Hopf algebra are not determined by its tensor category of representations.
\end{abstract}

\section{Introduction and Main Results}

Let $G$ be a finite group. The character table of $G$ provides substantial information about the group $G$ itself like, for instance, normal subgroups and their orders, the center $Z(G)$, the nilpotence or solvability, etc. In particular, whether $G$ is simple (respectively, solvable) or not can be established by inspection of its character table [10]. The character table provides the same information about $G$ as does the Grothendieck ring of the tensor category $\operatorname{Rep} G$ [3]. The above information is thus $a$ fortiori determined by $\operatorname{Rep} G$.

Finite dimensional Hopf algebras with tensor equivalent categories of representations are obtained from one another by a twisting deformation. Properties of $H$ invariant under twisting are of special interest because they depend only on the tensor category $\operatorname{Rep} H$.

In this paper we give a series of examples showing that the notions of simplicity and (semi)solvability of a (semisimple) Hopf algebra are not twist invariants; that is, they are not categorical notions.

In a first part we show that certain twists of the symmetric group $\mathbb{S}_{n}$ on $n$ letters are simple as Hopf algebras, for $n \geq 5$ (Theorem 3.5). To prove this we give a necessary condition for a group-like element in the dual of a twisting of a finite group to be central, in the case where the twisting is 'lifted' from an abelian subgroup.

Let $p, r$ and $q$ be prime numbers such that $q$ divides $p-1$ and $r-1$. In a second part we show that a family of supersolvable groups $G$ of order $p r q^{2}$ can be deformed through a twist into nontrivial simple Hopf algebras (Theorem 4.5). These twists are also lifted from an abelian subgroup of $G$. The proof relies on the comparison of the (co)representation theory of the given twistings [7] with that of an extension [12]. We

Received by the editors November 3, 2006.

1991 Mathematics Subject Classification. 16W30.

This work was partially supported by CONICET, Fundación Antorchas, Agencia Córdoba Ciencia, ANPCyT and Secyt (UNC). 
also make use of the classification of semisimple Hopf algebras in dimension $p$ and $p q$ $[26,14,6,9]$.

It is known that a semisimple Hopf algebra of dimension $p^{n}$ is always semisolvable $[13,12]$. On the other hand, we prove that if the group $G$ is nilpotent then any twisting of $k G$ is semisolvable.

Our results imply:

(a) There exists a simple semisimple Hopf algebra which is neither twist equivalent to a simple group nor to the dual of a simple group.

This answers negatively Question 2.3 in [1].

(b) There exists a semisimple Hopf algebra of dimension $p^{2} q^{2}$ which is simple as Hopf algebra.

Therefore the analogue of Burnside's $p^{a} q^{b}$-Theorem for finite groups does not hold for semisimple Hopf algebras. This concerns an open question raised by S. Montgomery; see [1, Question 4.17].

(c) There exists a nontrivial semisimple Hopf algebra which is simple in dimension 36.

This example gives a negative answer to [11, Question, pp. 269]. In dimension $<60$ this is the only possible such Hopf algebra, by [16].

(d) There exists a semisimple Hopf algebra which is a bosonization but not an extension.

This answers Question 2.13 of [1]. Indeed, the Hopf algebras in Theorem 4.5 can be built up as a Majid-Radford biproduct or bosonization.

We show that there are exactly two twistings of groups of order 60 that can be simple as Hopf algebras: the twisting of $\mathbb{A}_{5}$ constructed in [19], and the (self-dual) twisting of $D_{3} \times D_{5}$ discussed in Subsection 4.2. This contributes to the problem in [1, Question 2.4].

The paper is organized as follows. In Section 2 we recall known facts on normal Hopf subalgebras and the twisting construction. In Section 3 we prove some results on dual central group-like elements in twisting of finite groups, and present the construction for the symmetric groups. Section 4 contains the construction for a family of supersolvable groups; at the end of this section we state our results in dimensions 60 and 36. Finally, in Section 5 we discuss twisting of nilpotent groups.

Along this paper we shall work over an algebraically closed base field $k$ of characteristic zero. The notation for Hopf algebras is standard: $\Delta, \epsilon, S$, denote the comultiplication, counit and antipode, respectively.

\section{Preliminaries}

We discuss in this section basic facts on normal Hopf subalgebras and twisting deformations.

2.1. Normal Hopf subalgebras. Let $A$ be a finite dimensional Hopf algebra over $k$. The (left) adjoint action of $A$ on itself is defined by ${ }^{h} x=\sum h_{1} x\left(S h_{2}\right), x, h \in A$. A Hopf subalgebra $K \subseteq A$ is called normal if it is stable under the adjoint action; $A$ is called simple if it contains no proper normal Hopf subalgebras. Dualizing the 
notion of normal Hopf subalgebra, we obtain the notion of conormal quotient Hopf algebra. The notion of simplicity is self-dual; that is, $A$ is simple if and only if $A^{*}$ is.

Let $K \subseteq A$ be a normal Hopf subalgebra. Then $B=A / A K^{+}$is a conormal quotient Hopf algebra and the sequence of Hopf algebra maps $k \longrightarrow K \longrightarrow A \longrightarrow B \longrightarrow k$ is an exact sequence of Hopf algebras. In this case we shall say that $A$ is an extension of $B$ by $K$. As a Hopf algebra, $A$ is isomorphic to a bicrossed product $A \cong K^{\tau} \#_{\sigma} B$.

We recall the following results for future use.

Proposition 2.1. [16, Corollary 1.4.3]. Let $K \subseteq A$ be a normal Hopf subalgebra. Suppose that $\operatorname{dim} K$ is the least prime number dividing $\operatorname{dim} A$. Then $K$ is central in $A$.

Corollary 2.2. Let $\pi: A \rightarrow B$ be a conormal quotient Hopf algebra. Suppose that $\operatorname{dim} B$ is the least prime number dividing $\operatorname{dim} A$. Then $G\left(B^{*}\right) \subset Z\left(A^{*}\right) \cap G\left(A^{*}\right)$.

Example 2.3. Let $G$ be a finite group. The normal Hopf subalgebras of $k G$ are of the form $k H$ where $H$ is a normal subgroup of $G$. In particular, $k G$ is simple as a Hopf algebra if and only if $G$ is a simple group. In addition, if $k G$ is simple, then it possesses no nontrivial quotient Hopf algebra. Therefore $k^{G}$ contains no proper Hopf subalgebra.

2.2. Twisting. Let $A$ be a finite dimensional Hopf algebra. The category Rep $A$ of its finite dimensional representations is a finite tensor category with tensor product given by the diagonal action of $A$ and unit object $k$.

Finite tensor categories of the form $\operatorname{Rep} A$ are characterized, using tannakian reconstruction arguments, as those possessing a fiber functor with values in the category of vector spaces over $k$. The forgetful functor $\operatorname{Rep} A \rightarrow \mathrm{Vec}_{k}$ is a fiber functor and other fiber functors correspond to twisting the comultiplication of $A$ in the following sense.

Definition 2.4. [4]. A twist in $A$ is an invertible $J \in A \otimes A$ satisfying:

$$
\begin{aligned}
(\Delta \otimes i d)(J)(J \otimes 1) & =(i d \otimes \Delta)(J)(1 \otimes J), \\
(\varepsilon \otimes i d)(J) & =(i d \otimes \varepsilon)(J)=1 .
\end{aligned}
$$

If $J \in A \otimes A$ is a twist, $\left(A^{J}, m, \Delta^{J}, \varepsilon, S^{J}\right)$ is a Hopf algebra with $A^{J}=A, \Delta^{J}(h)=$ $J^{-1} \Delta(h) J$, and $S^{J}(h)=v^{-1} S(h) v, h \in A, v=m \circ(S \otimes i d)(J)$.

The Hopf algebras $A$ and $A^{\prime}$ are called twist equivalent if $A^{\prime} \cong A^{J}$. It is known that $A$ and $A^{\prime}$ are twist equivalent if and only if $\operatorname{Rep} A \cong \operatorname{Rep} A^{\prime}$ as tensor categories [23]. Therefore, properties like (quasi)triangularity, semisimplicity or the structure of the Grothendieck ring are preserved under twisting deformation.

Remark 2.5. Let $\pi: A \rightarrow B$ be a Hopf algebra map and let $J \in A \otimes A$ be a twist. Then $(\pi \otimes \pi)(J)$ is a twist for $B$ and $\pi: A^{J} \rightarrow B^{(\pi \otimes \pi)(J)}$ is a Hopf algebra map.

Note that if $J \in K \otimes K$ is a twist for the Hopf subalgebra $K \subset A$, then $J \in A \otimes A$ is also a twist for $A$. We shall say that such $J$ is lifted from the Hopf subalgebra $K$ $[5,25]$. 
If $A=k G$ is a group algebra, with $G$ a finite abelian group, the (gauge) equivalence classes of twists for $A$ are in bijective correspondence with the group $H^{2}\left(G, k^{*}\right)$ [15, Proposition 3]. The twist $J$ corresponding to the cocycle $\omega: G \times G \rightarrow k^{*}$ is given by

$$
J=\sum_{\alpha, \beta \in \widehat{G}} \omega(\alpha, \beta) e_{\alpha} \otimes e_{\beta},
$$

where $e_{\chi}=\frac{1}{|G|} \sum_{h \in G} \chi\left(h^{-1}\right) h, \chi \in \widehat{G}$, is a basis of orthogonal central idempotents of $k G$.

Twists in finite groups have been completely classified [15, 8]. Every twist in $k G$ is lifted from a minimal subgroup $H \subseteq G$ for $J$; that is, the components of $J_{21}^{-1} J$ span $k H$. Gauge equivalence classes of twists are classified by classes of pairs $(H, \omega)$, where $H$ is some solvable subgroup whose order is a square, and $\omega \in H^{2}\left(H, k^{*}\right)$ is a non-degenerate 2-cocycle on $H$.

When $H$ is abelian, the twist corresponding to $(H, \omega)$ is given by $(2.3)$.

Recall that an element $g \in H$ is called $\omega$-regular if $\omega(g, h)=\omega(h, g)$, for all $h \in Z_{H}(g)$ (this definition depends only on the class of $g$ under conjugation). Then the cocycle $\omega \in H^{2}\left(H, k^{*}\right)$ is non-degenerate if and only if $\{1\}$ is the only $\omega$-regular class in $H$.

In particular, if $H$ is abelian, the cocycle $\omega$ is non-degenerate if and only if the skew-symmetric bilinear form $\omega_{21}^{-1} \omega: H \times H \rightarrow k^{*},(g, h) \mapsto \omega(g, h) \omega^{-1}(h, g)$, is non-degenerate.

The following lemma follows from [7]. See also [19, Lemma 2.11].

Lemma 2.6. Let $J \in k G \otimes k G$ be the twist associated to the pair $(H, \omega)$, where $H$ is the minimal subgroup of $J$. Then $(k G)^{J}$ is cocommutative if and only if $H \unlhd G, H$ is abelian and $\omega$ is ad $G$-invariant in $H^{2}\left(H, k^{*}\right)$.

In particular, every nonsymmetric twist lifted from an abelian subgroup of a simple nonabelian group, or from an abelian subgroup not containing normal subgroups of $G$, gives rise to a noncocommutative Hopf algebra.

Example 2.7. [19]. Let $\mathbb{A}_{n}$ be the alternating group in $n$ elements, $n \geq 4$. Consider the subgroup $H \cong \mathbb{Z}_{2} \times \mathbb{Z}_{2}$ generated by $a=(12)(34)$ and $b=(13)(24)$. Let $\omega$ be a 2 -cocycle whose cohomology class is nontrivial. Since $\omega$ is not symmetric, by Lemma 2.6, $A=\left(k \mathbb{A}_{n}\right)^{J}$ is a simple noncommutative and noncocommutative Hopf algebra, for all $n \geq 5$.

As an algebra, $\left(k \mathbb{A}_{5}\right)^{J} \cong k \times M_{3}(k)^{(2)} \times M_{4}(k) \times M_{5}(k)$.

Recall that there is a one to one correspondence between quotient Hopf algebras of $H$ and hereditary subrings of the Grothendieck ring $K_{0}(H)$ [17]. The subring corresponding to the quotient $H \rightarrow \bar{H}$ is $K_{0}(\bar{H}) \subseteq K_{0}(H)$.

Proposition 2.8. [19]. Let $G$ be a finite simple group and let $J \in k G \otimes k G$ be a twist. Then $(k G)^{J}$ is simple as a Hopf algebra.

Indeed, the result is true under the weaker assumption that $J$ is a pseudo-twist in $k G$. We shall see that the converse of Proposition 2.8 is not true. 


\section{Simple deformations of the symmetric groups}

Let $(A, R)$ be a finite dimensional quasitriangular Hopf algebra. The map $f$ : $G\left(A^{*}\right) \rightarrow G(A)$, given by $f(\eta)=R^{(1)} \eta\left(R^{(2)}\right)$, where $R=R^{(1)} \otimes R^{(2)}$, is an antihomomorphism of groups. In addition, $\eta \in G\left(A^{*}\right)$ is central in $A^{*}$ if and only if $f(\eta)$ is central in $A[20$, Proposition 3].

In what follows we shall consider a finite group $G$ and an abelian subgroup $H \subseteq G$. Let $A=(k G)^{J}$, where $J \in k G \otimes k G$ is a twist lifted from $H$, written in the form (2.3). Observe that $G\left(A^{*}\right)=\widehat{G}$.

Theorem 3.1. Suppose that $Z(G)=1$. Let $\eta \in \widehat{G}$. Then $\eta \in G\left(A^{*}\right) \cap Z\left(A^{*}\right)$ if and only if $\left.\eta\right|_{H}$ is $\omega$-regular.

If $\omega$ is non-degenerate, $\eta \in G\left(A^{*}\right) \cap Z\left(A^{*}\right)$ if and only if $\left.\eta\right|_{H}=1$.

Here, $\left.\eta\right|_{H}$ is the restriction of $\eta$ to $H$.

Proof. The Hopf algebra $A$ is triangular with $R$-matrix

$$
R=J_{21}^{-1} J=\sum_{\alpha, \beta \in \widehat{H}} \omega(\alpha, \beta) \omega^{-1}(\beta, \alpha) e_{\alpha} \otimes e_{\beta} .
$$

Since $Z(G)=1$, we have $Z(A) \cap G(A)=1$. That is, $\eta \in G\left(A^{*}\right) \cap Z\left(A^{*}\right)$ if and only if $f(\eta)=1$. Let $\eta \in G\left(A^{*}\right)$. By the orthogonality relations, $\eta\left(e_{\chi}\right)=\delta_{\chi,\left.\eta\right|_{H}}$, for all $\chi \in \widehat{H}$. Therefore,

$$
f(\eta)=\sum_{\alpha, \beta \in \widehat{H}} \omega(\alpha, \beta) \omega^{-1}(\beta, \alpha) e_{\alpha} \eta\left(e_{\beta}\right)=\sum_{\alpha \in \widehat{H}} \omega\left(\alpha,\left.\eta\right|_{H}\right) \omega^{-1}\left(\left.\eta\right|_{H}, \alpha\right) e_{\alpha} .
$$

Then $f(\eta)=1$ if and only if $\omega\left(\chi,\left.\eta\right|_{H}\right) \omega^{-1}\left(\left.\eta\right|_{H}, \chi\right)=1, \forall \chi \in \widehat{H}$.

Corollary 3.2. Suppose $Z(G)=1$ and $\omega$ is non-degenerate. Then the order of $H$ divides $\left[A^{*}: G\left(A^{*}\right) \cap Z\left(A^{*}\right)\right]$.

Proof. In this case, $\left.\eta\right|_{H}=1$, for all $\eta \in G\left(A^{*}\right) \cap Z\left(A^{*}\right)$, by Theorem 3.1. Then the projection $A \rightarrow k\left(G\left(A^{*}\right) \cap Z\left(A^{*}\right)\right)^{*}$ restricts trivially to $H$. The corollary follows from [18].

Let $\pi: A \rightarrow k F$ be a Hopf algebra quotient with $F$ an abelian group. Then the group $\widehat{F} \cong F$ can be identified with a subgroup of $G\left(A^{*}\right)$.

Theorem 3.3. Suppose $Z(G)=1$. Let $\pi: A \rightarrow k F$ be a quotient Hopf algebra, where $F \cong \mathbb{Z}_{p}$ and $p$ is the least prime dividing $|G|$. Then $\pi$ is normal if and only if $\left.\mu\right|_{H}$ is $\omega$-regular, for all $\mu \in \widehat{F}$. Assume $\omega$ is non-degenerate. Then $\pi$ is normal if and only if $\left.\mu\right|_{H}=1$, for all $\mu \in \widehat{F}$.

Proof. By Theorem 3.1, condition $\omega\left(\chi,\left.\mu\right|_{H}\right)=\omega\left(\left.\mu\right|_{H}, \chi\right)$, for all $\chi \in \widehat{H}, \mu \in \widehat{F}$, is equivalent to $\widehat{F} \subset Z\left(A^{*}\right) \cap G\left(A^{*}\right)$. In view of Proposition 2.2 this is equivalent to $\pi$ being normal.

Let $\pi: \mathbb{S}_{n} \rightarrow \mathbb{Z}_{2}$ be the only nontrivial epimorphism. So that ker $\pi=\mathbb{A}_{n}$. Let $H \subseteq \mathbb{S}_{n}$ be an abelian subgroup, and let $A=\left(k \mathbb{S}_{n}\right)^{J}$ be a twisting with $J \in k H \otimes k H$ given by (2.3). As noted in Remark 2.5, $\pi: A \rightarrow k \mathbb{Z}_{2}$ is a Hopf algebra map.

Consider the sign representation $\sigma: \mathbb{S}_{n} \rightarrow k^{*}$. 
Corollary 3.4. $\pi: A \rightarrow k \mathbb{Z}_{2}$ is normal if and only if $\left.\sigma\right|_{H}$ is $\omega$-regular.

Assume $\omega$ is non-degenerate. Then $\pi$ is normal if and only if $H \subseteq \mathbb{A}_{n}$.

Let $n \geq 4$. Consider the abelian subgroup $H=<t_{1}, t_{2}>\cong \mathbb{Z}_{2} \times \mathbb{Z}_{2}$ of $\mathbb{S}_{n}$, generated by the transpositions $t_{1}=(12), t_{2}=(34)$.

We have $\widehat{H}=<a_{1}, a_{2}>$, where $a_{i}\left(t_{j}\right)=1$ if $i \neq j$, and $a_{i}\left(t_{i}\right)=-1$.

Let $\omega$ be the unique nontrivial cocycle on $\widehat{H}$ (up to coboundaries). Then $\omega$ is non-degenerate. Let $J \in k H^{\otimes 2}$ be the corresponding twist.

Theorem 3.5. Suppose $n \geq 5$. Then $\left(k \mathbb{S}_{n}\right)^{J}$ is simple.

Proof. In this case $\pi: \mathbb{S}_{n} \rightarrow \mathbb{Z}_{2}$ is the only nontrivial quotient of $\mathbb{S}_{n}$. Since twisting preserves Grothendieck rings, $A=\left(k \mathbb{S}_{n}\right)^{J}$ has also a unique nontrivial quotient $\pi$ : $A \rightarrow k \mathbb{Z}_{2}$. Since $H \nsubseteq \mathbb{A}_{n}$, by Corollary $3.4, \pi$ is not normal. Therefore $\left(k \mathbb{S}_{n}\right)^{J}$ is simple, as claimed.

Remark 3.6. Let $A=\left(k \mathbb{S}_{n}\right)^{J}$ as in Theorem 3.5. Then $A^{*}$ is simple and it is not a twisting of any group. Otherwise $A^{*}$ and $A$ would be both quasitriangular and simple, hence $G(A) \cong G\left(A^{*}\right)[21$, Proposition 4]. But this is not the case, since $H \subseteq G(A)$ and $\left|G\left(A^{*}\right)\right|=2$.

Another family of examples arises from the construction in [2]. Let $t_{i}$ denote the transposition $(2 i-1,2 i) \in \mathbb{S}_{2 n}, 1 \leq i \leq n$. Consider the abelian subgroup $H=<t_{i}, 1 \leq i \leq n>\cong\left(\mathbb{Z}_{2}\right)^{n}$ of $\mathbb{S}_{2 n}$.

We have $\widehat{H}=<a_{i}: 1 \leq i \leq n>$, where $a_{i}\left(t_{j}\right)=1$ if $i \neq j$, and $a_{i}\left(t_{i}\right)=-1$. Note that $\left.\sigma\right|_{H}=a_{1} a_{2} \cdots a_{n}$.

Consider the bicharacter $\omega: \widehat{H} \times \widehat{H} \rightarrow k^{*}, \omega\left(a_{i}, a_{j}\right)=-1, i<j, \omega\left(a_{i}, a_{j}\right)=1$, $i \geq j$. This example does not fulfill the condition in Proposition 3.4 for $n \geq 2$ even and $a=a_{1}$, since we have $\omega\left(\left.\sigma\right|_{H}, a_{1}\right)=(-1)^{n-1}=-1$, while $\omega\left(a_{1},\left.\sigma\right|_{H}\right)=1$. Let $J$ be the corresponding twist.

Theorem 3.7. Suppose $n \geq 3, n$ even. Then $\left(k \mathbb{S}_{2 n}\right)^{J}$ is simple.

Proof. Again in this case $\pi: \mathbb{S}_{2 n} \rightarrow \mathbb{Z}_{2}$ is the only nontrivial quotient of $\mathbb{S}_{2 n}$. So that $A=\left(k \mathbb{S}_{2 n}\right)^{J}$ has also a unique nontrivial quotient $\pi: A \rightarrow k \mathbb{Z}_{2}$. By Theorem $3.5 \pi$ is not normal. Thus $\left(k \mathbb{S}_{2 n}\right)^{J}$ is simple.

Remark 3.8. Let us see that for any twist $J \in k \mathbb{S}_{4} \otimes k \mathbb{S}_{4},\left(k \mathbb{S}_{4}\right)^{J}$ is not simple; see [16, Chapter 6]. We know from $[15,8]$ that the minimal subgroup $H$ for $J$ is some solvable subgroup whose order is a square admitting a non-degenerate 2-cocycle. Then for $k \mathbb{S}_{4}$ a twist must be lifted from a subgroup $H$ of order 4 isomorphic to $\mathbb{Z}_{2} \times \mathbb{Z}_{2}$.

Claim 3.9. Let $A=\left(k \mathbb{S}_{4}\right)^{J}$, where $J$ is the twist lifted from a subgroup $H \cong \mathbb{Z}_{2} \times \mathbb{Z}_{2}$ which is not normal and $\omega \neq 1$. Then $G(A) \cong D_{4}$.

Proof. Let $D \cong D_{4}$ be the Sylow 2-subgroup of $\mathbb{S}_{4}$ containing $H$. Then there are Hopf algebra inclusions $k H \cong(k H)^{J} \hookrightarrow(k D)^{J} \hookrightarrow\left(k \mathbb{S}_{4}\right)^{J}$.

Since $(k D)^{J}$ is not commutative and of dimension $8,(k D)^{J} \cong k D$ as Hopf algebras [24]. Thus 8 divides $\left|G\left(\left(k \mathbb{S}_{4}\right)^{J}\right)\right|$. As $H$ contains no subgroup which is normal in $\mathbb{S}_{4}$, by Lemma $2.6,\left(k \mathbb{S}_{4}\right)^{J}$ is not cocommutative. Then $\left|G\left(\left(k \mathbb{S}_{4}\right)^{J}\right)\right|=8$ and $G(A) \cong D \cong$ $D_{4}$. 
Let the quotients $B=\mathbb{S}_{4} / K \cong \mathbb{S}_{3}$, and $\zeta:\left(k \mathbb{S}_{4}\right)^{J} \rightarrow\left(k \mathbb{S}_{3}\right)^{\zeta(J)} \cong k \mathbb{S}_{3}$. We claim that $\zeta$ is normal.

Indeed, we have $\operatorname{dim} A^{\operatorname{coB}}=4$. Then $\operatorname{dim} A^{\operatorname{coB}} \cap k G(A)=\operatorname{dim} k G(A)^{c o B}=1,2,4$, since $|k G(A)|=8$. Also, $\operatorname{dim} k G(A)^{\operatorname{co} B} \operatorname{dim} \zeta(k G(A))=8$. If $\operatorname{dim} k G(A)^{\operatorname{coB}}=1,2$, then $\operatorname{dim} \zeta(k G(A))=8$, 4, which is impossible. Then $\operatorname{dim} A^{\operatorname{coB}} \cap k G(A)=4$; that is, $A^{c o B} \subset k G(A)$. Hence $A^{c o B}$ is a normal Hopf subalgebra.

\section{Deformations of a family of supersolvable groups}

Let $p, q$ and $r$ be prime numbers such that $q$ divides $p-1$ and $r-1$. Let $G_{1}=\mathbb{Z}_{p} \rtimes \mathbb{Z}_{q}$ and $G_{2}=\mathbb{Z}_{r} \rtimes \mathbb{Z}_{q}$ be the only nonabelian groups of orders $p q$ and $r q$, respectively. Let $G=G_{1} \times G_{2}$ and let $\mathbb{Z}_{q} \times \mathbb{Z}_{q} \cong H \subseteq G$ a subgroup of order $q^{2}$. In particular, $G$ is supersolvable and $Z(G)=1$.

Let $1 \neq \omega \in H^{2}\left(\widehat{H}, k^{*}\right), J \in k G \otimes k G$ the twist lifted from $H$ corresponding to $\omega$. Let also $A=(k G)^{J}$. Note that the cocycle $\omega$ is nondegenerate. Also, $A$ is a nontrivial Hopf algebra of dimension $p r q^{2}$.

Lemma 4.1. $A \cong k^{\left(q^{2}\right)} \times M_{q}(k) \times \cdots \times M_{q}(k) \times M_{q^{2}}(k) \times \cdots \times M_{q^{2}}(k)$ as an algebra.

Proof. As an algebra, $A=k G \simeq k G_{1} \otimes k G_{2}$.

The coalgebra structure of $A$ follows from the result in [7] on representations of cotriangular semisimple Hopf algebras. In particular:

Lemma 4.2. $G(A) \cong H$ is of order $q^{2}$.

Proof. Let, for all $g \in G, H_{g}=H \cap g H g^{-1}$, and let $\widetilde{\omega}$ be the 2-cocycle on $H_{g}$ given by $\widetilde{\omega}(x, y)=\omega^{-1}\left(g^{-1} x g, g^{-1} y g\right) \omega(x, y)$. By [7] the irreducible representations of $A^{*}$ are classified by pairs $(\bar{g}, X)$, where $\bar{g} \in H \backslash G / H$ is a double coset modulo $H$ and $X$ is an irreducible representation of the twisted group algebra $k_{\widetilde{\omega}} H_{g}$. The dimension of the representation $W_{\bar{g}, X}$ corresponding to $(\bar{g}, X)$ is $\operatorname{dim} W_{\bar{g}, X}=\left[H: H_{g}\right] \operatorname{dim} X$.

Note that $\widetilde{\omega}$ is trivial on $H_{1}=H$. Thus $\operatorname{dim} W_{\overline{1}, X}=1$, for all possible choices of $X$, giving $|H|$ distinct one-dimensional representations.

Also, $\operatorname{dim} W_{\bar{g}, X}=1$ if and only if $H_{g}=H$ and $\operatorname{dim} X=1$. That is, if and only if $g \in N_{G}(H)$ and $\operatorname{dim} X=1$. In our example $N_{G}(H)=H$. So if $\operatorname{dim} W_{\bar{g}, X}=1$, then $\bar{g}=\overline{1}$ and there are no more one-dimensional representations.

Lemma 4.3. Suppose $A$ is not simple and let $K \subseteq A$ be a proper normal Hopf subalgebra. Then $\operatorname{dim} K=p q$ or $r q$.

Moreover, $K$ is necessarily commutative and not cocommutative and there is an exact sequence of one of the forms

$$
k \rightarrow k^{G_{1}} \rightarrow A \rightarrow k G_{2} \rightarrow k, \quad k \rightarrow k^{G_{2}} \rightarrow A \rightarrow k G_{1} \rightarrow k .
$$

Proof. Let $B=A / A K^{+}$. There is a Hopf algebra inclusion $B^{*} \subseteq A^{*}$ and $B^{*}$ is normal in $A^{*}$. Since $Z(G)=1$, we have $G(A) \cap Z(A)=1$. In particular, $\operatorname{dim} K \neq q[26]$. Suppose $\operatorname{dim} B^{*}=q$. Then $B^{*}=k G\left(B^{*}\right)$ and $G\left(B^{*}\right) \subseteq G\left(A^{*}\right) \cap Z\left(A^{*}\right)$. Since $\omega$ is non-degenerate, Corollary 3.2 implies that $q^{2} \mid\left[A^{*}: B^{*}\right]=\operatorname{prq}$, which is impossible. Hence $\operatorname{dim} B \neq q$. 
If $\operatorname{dim} B=q^{2} p, q^{2} r, q^{2}$, then $\operatorname{dim} K=r, p, p r$ and $K$ is a group algebra or a dual group algebra $[26,14,6,9]$. Thus $A$ has group-like elements of order $p$ or $r$, contradicting $|G(A)|=q^{2}$. Then $\operatorname{dim} K=p q, r q$. Since $|G(A)|=q^{2}, K \cong k^{G_{1}}$ or $k^{G_{2}}$. Similarly, these are the only possibilities for $B^{*}$.

Remark 4.4. The results in [7] actually imply that $A^{*} \cong A$ as algebras. Once we have established that $A$ is simple, then we will see in Subsection 4.1 that $A^{* \operatorname{cop}} \cong A$ as Hopf algebras.

Theorem 4.5. A is simple as a Hopf algebra.

Proof. Suppose not. We shall compute the dimensions of the irreducible $A$-modules to get a contradiction. By Lemma 4.3, without loss of generality, we may assume that $A$ is a bicrossed product $A \cong k^{G_{1} \tau} \#_{\sigma} k G_{2}$. By [12] the irreducible $A$-modules are classified by pairs $(x, U)$, where $x$ is a representative of an orbit of the action of $G_{2}$ in $G_{1}$ and $U$ is an irreducible projective representation of the stabilizer $\left(G_{2}\right)_{x}$ of $x$. The irreducible module $W_{(x, U)}$ corresponding to $(x, U)$ is the induced module $W_{(x, U)}=\operatorname{Ind}_{B}^{A}(k x \otimes U)$, where $B=k^{G_{1}} \#_{\sigma}\left(k G_{2}\right)_{x}$. Therefore $\operatorname{dim} W_{(x, U)}=\left[G_{2}:\right.$ $\left.\left(G_{2}\right)_{x}\right] \operatorname{dim} U$.

Hence the dimension of an irreducible module cannot be $q^{2}$. This contradicts Lemma 4.1. The theorem is now established.

Remark 4.6. We point out the following consequence of Theorem 4.5. By Lemmas 4.1 and 4.2, $A$ is a biproduct $A=R \# k H$ in the sense of Majid-Radford, with $\operatorname{dim} R=p r$. This gives a nontrivial braided Hopf algebra structure on $R$ over $H$, in such a way that the corresponding biproduct is simple. This answers [1, Question 2.13].

Letting $p=r$ in Theorem 4.5 we obtain:

Theorem 4.7. Let $p, q$, be prime numbers such that $q \mid p-1$. Then there exists a semisimple Hopf algebra of dimension $p^{2} q^{2}$ which is simple as a Hopf algebra.

The theorem disproves a conjectured 'quantum version' of Burnside's $p^{a} q^{b}$-Theorem in the context of semisimple Hopf algebras. It also gives a negative answer to $[1$, Question 2.3].

4.1. Self-duality. If $G$ is finite group and $J$ is a minimal twist in $G$, then the twisted group algebra $A=(k G)^{J}$ satisfies $A \cong A^{* \operatorname{cop}}$ [8]. We show next that this is also true under other restrictions on $G$ and $J$.

Proposition 4.8. Let $G$ be a finite solvable group and let $J \in k G \otimes k G$ be any twist. Assume $A=(k G)^{J}$ is simple. Then $A \cong A^{* c o p}$.

By comparing the descriptions of the representation theories of $G$ and $\left(k G^{J}\right)^{*}$, we see that this proposition imposes severe restrictions on the possible groups $G$ satisfying the assumptions.

Proof. Since $A$ is simple, then $Z(G)=1$. Suppose $F \unlhd G$. Then $G$ acts on $k^{F}$ by the adjoint action and the smash product $k^{F} \# k G$ is a Hopf algebra quotient of $D(G)$ (actually, $D(G)$ corresponds to $F=G$ ). For the Hopf algebra $D_{F}(G)=k^{F} \# k G$ we have $G\left(D_{F}(G)\right) \cap Z\left(D_{F}(G)\right)=\widehat{F} \times Z(G)=\widehat{F}$ and

$$
D_{F}(G) / D_{F}(G)(k \widehat{F})^{+} \cong k^{[F, F]} \# k G=D_{[F, F]}(G) .
$$


Denote $G=G^{(0)}, G^{(i+1)}=\left[G^{(i)}, G^{(i)}\right], i \geq 0$. Since $G$ is solvable, there exists $m \geq 1$ such that $G^{(m)}=1$. Iterating the construction above, we get a sequence

$$
D(G) \stackrel{\pi_{1}}{\rightarrow} D_{G^{(1)}}(G) \stackrel{\pi_{2}}{\rightarrow} D_{G^{(2)}}(G) \stackrel{\pi_{3}}{\rightarrow} \ldots \stackrel{\pi_{m}}{\rightarrow} D_{G^{(m)}}(G)=k G
$$

where every map $\pi_{i}$ has central kernel. Since $A$ is twist equivalent to $k G$ then $D(A)$ is twist equivalent to $D(G)$. In view of Lemma 5.2, we get another sequence of Hopf algebra maps with central kernels

$$
D(A) \stackrel{\pi_{1}}{\rightarrow} K_{1} \stackrel{\pi_{2}}{\rightarrow} K_{2} \stackrel{\pi_{3}}{\rightarrow} \cdots \stackrel{\pi_{m}}{\rightarrow} K_{m}=(k G)^{J^{\prime}} .
$$

Since the maps $\pi_{i}$ are normal and $A$ and $A^{* \text { cop }}$ are simple and nontrivial, we may assume that the composition $\pi_{1} \circ \cdots \circ \pi_{m}: D(A) \rightarrow(k G)^{J^{\prime}}$ is injective when restricted to $A$ and to $A^{* \text { cop }}$. By dimension, $A \cong(k G)^{J^{\prime}} \cong A^{* c o p}$.

4.2. Twisting deformations of groups of order 60. As we saw in Example 2.7, there is a nontrivial simple Hopf algebra of dimension 60, obtained as a twisting of the alternating group $\mathbb{A}_{5}$. Another example arises as a twisting deformation of the group

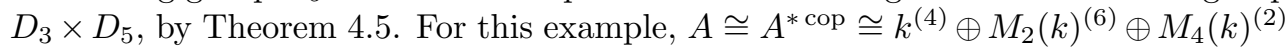
as algebras; so $A$ is not a twisting of $k \mathbb{A}_{5}$. We shall prove that these are the only simple Hopf algebras that can arise as twistings of groups of order 60 .

For the rest of this subsection, $G$ will be a group of order $60, J \in k G \otimes k G$ a twist, and $A=(k G)^{J}$ a nontrivial deformation. Since $A$ is not trivial, the minimal subgroup $H$ associated to $J$ must be of order 4 . Then necessarily $H \cong \mathbb{Z}_{2} \times \mathbb{Z}_{2}$ and $J$ corresponds to the only nontrivial 2-cocycle on $H$.

We assume first that $G$ is not simple.

Lemma 4.9. Suppose $A$ is simple. Then $G \cong D_{3} \times D_{5}$.

Proof. First note that since $G$ is not simple, then $G$ contains a unique subgroup of order 5. Second, the subgroup $H$ cannot be contained in a normal subgroup $P$ of $G$, since otherwise, the Hopf subalgebra $k P^{J} \subset(k G)^{J}$ would be normal. Similarly, we may assume that $Z(G)=1$.

Let $S \unlhd G$ of order 5 . We may assume that $G^{\prime}=G / S$ has a normal subgroup $T$ of order 3, since otherwise $G$ would contain a normal subgroup of order 20, thus containing $H$. Then $N=\pi^{-1}(T) \unlhd G$ is of order 15 ; so $G$ is a semidirect product $N \rtimes H$. Because $Z(G)=1, G \cong D_{3} \times D_{5}$.

Theorem 4.10. Let $|G|=60$ and let $J \in k G \otimes k G$ be a twist such that $A=(k G)^{J}$ is not cocommutative and simple. Then either

(i) $G=\mathbb{A}_{5}$ and $A$ is isomorphic to the Hopf algebra in Example 2.7; or

(ii) $G=D_{3} \times D_{5}$ and $A$ is isomorphic to the self-dual Hopf algebra in Theorem 4.5.

Since the Hopf algebra in (i) is not self-dual, this gives three simple examples of semisimple Hopf algebras in dimension 60.

Proof. We use Lemma 4.9. Note that the subgroups of order 4 in $G$ are conjugated, and $\left|H^{2}\left(\mathbb{Z}_{2} \times \mathbb{Z}_{2}, k^{*}\right)\right|=2$. Hence every pair of nontrivial twists in $G$ gives rise to isomorphic Hopf algebras. 
4.3. Twisting deformations of groups of order 36 . The main construction of this section gives an example of a nontrivial semisimple Hopf algebra $A$ of dimension 36 that is simple as a Hopf algebra. This is thus the smallest semisimple Hopf algebra which is not semisolvable and the unique simple case in dimension 36 [16]. The Hopf algebra $A$ is a twisting of the group $D_{3} \times D_{3}$, and we have $A \cong A^{* \text { cop } \cong}$ $k^{(4)} \oplus M_{2}(k)^{(4)} \oplus M_{4}(k)$ as algebras.

Theorem 4.11. Let $G$ be a group of order 36 and let $J \in k G \otimes k G$ be a twist such that $(k G)^{J}$ is simple. Then $G \cong D_{3} \times D_{3}$ and $(k G)^{J} \cong A$.

Proof. We may assume $Z(G)=1$. If $J$ is a non-degenerate twist, then necessarily $G=\mathbb{Z}_{3} \times \mathbb{A}_{4}$ or $G=\mathbb{Z}_{2} \ltimes\left(\mathbb{Z}_{3} \times \mathbb{Z}_{6}\right)$. This contradicts the assumption $Z(G)=1$; hence, we may assume that $J$ is not minimal. Moreover, the minimal subgroup $H$ of $G$ has order 4 or 9 and it is not cyclic. Also, $H$ is not contained in any normal subgroup. This leads to consider the case $H \simeq \mathbb{Z}_{2} \times \mathbb{Z}_{2}$ and $G \cong D_{3} \times D_{3}$. Moreover all subgroups of order 4 are conjugated and the conjugation action must preserve nontrivial twists.

\section{Twisting of nilpotent groups}

We have shown simple Hopf algebras obtained as twisting of supersolvable groups. We now prove that this cannot arise from nilpotent groups.

Let $H$ be a finite dimensional Hopf algebra over $k$.

Definition 5.1. [12]. A lower normal series for $H$ is a series of proper Hopf subalgebras $H_{n}=k \subset H_{n-1} \subset \cdots \subset H_{1} \subset H_{0}=H$, where $H_{i+1}$ is normal in $H_{i}$, for all $i$. The factors are the quotients $\bar{H}_{i}=H_{i} / H_{i} H_{i+1}^{+}$.

An upper normal series is inductively defined as follows. Let $H_{(0)}=H$. Let $H_{i}$ be a normal Hopf subalgebra of $H_{i-1}$ and define $H_{(i)}=H_{(i)} / H_{(i)} H_{i}^{+}$. Assume that $H_{n}=H_{(n-1)}$, for some positive integer $n$ such that $H_{(n)}=k$. The factors are the Hopf subalgebras $H_{i}$ of the quotients $H_{(i)}$.

Let $G$ be a finite group and let $A=(k G)^{J}$ be a twisting.

Lemma 5.2. Let $Z \subset G$ be a central subgroup. Then $k Z \subset A$ is a central Hopf subalgebra and $A / A(k Z)^{+} \cong(k G / Z)^{\bar{J}}$.

Proof. Since $A=k G$ as algebras, $k Z$ is central and $\Delta^{J}(a)=a \otimes a$. Let $\pi: k G^{J} \rightarrow$ $k(G / Z)^{\bar{J}}$ be the Hopf algebra map induced by the projection $G \rightarrow G / Z$. Since $k Z \subset A^{c o \pi}$ and $\operatorname{dim} A=\operatorname{dim} A^{c o \pi} \operatorname{dim} \pi(A), k Z=A^{c o \pi}$.

Theorem 5.3. Suppose $G$ is nilpotent. Then

(1) A has an upper normal series with factors $k \mathbb{Z}_{p}, p \mid \operatorname{dim} A$, prime.

(2) A has a lower normal series whose factors are cocommutative.

In particular, $A$ is semisolvable in the sense of [12].

Proof. (1). Since $G$ is nilpotent, $Z(G) \neq 1$. Let $Z \subset Z(G)$ be a subgroup of order $p$, $p$ prime. Let $H_{1}=k Z \cong k \mathbb{Z}_{p}$ and $H_{(1)}=A / A H_{1}^{+}$. By Lemma $5.2, H_{(1)} \cong k(G / Z)^{\frac{J}{J}}$. Since $G / Z$ is nilpotent, (1) follows by induction on $|G|$. 
(2). Let $H \subset G$ be the subgroup such that $J \in k H \otimes k H$ is minimal. Since every subgroup of a nilpotent group is subnormal [22], we have $H_{0}=H \triangleleft H_{1} \triangleleft \cdots \triangleleft$ $H_{n}=G$. Then

$$
k H_{0}=k H^{J} \triangleleft k H_{1}^{J} \triangleleft \cdots \triangleleft k H_{n}^{J}=k G^{J},
$$

is part of a lower normal series of $A$ with factors $k\left[H_{i+1} / H_{i}\right]$, since $J \in k H_{i}$, for all $i$. Since $H$ is nilpotent, there is an upper normal series $H_{(0)}=k H^{J} \rightarrow H_{(1)} \cdots \rightarrow$ $H_{(s)}=k$. Moreover, $k H^{J} \cong\left(k H^{J}\right)^{*}$ because $k H^{J}$ is minimal. Thus the dual of this series, that is, $k \hookrightarrow H_{(1)}^{*} \hookrightarrow \cdots \hookrightarrow\left(k H^{J}\right)^{*} \cong k H^{J}$, is a lower normal series for $k H^{J}$ that completes the series (5.1).

\section{Acknowledgement}

The authors thank N. Andruskiewitsch for stimulating discussions and encouragement.

\section{References}

[1] N. Andruskiewitsch, About finite dimensional Hopf algebras, Contemp. Math. 294 (2002), 1-57.

[2] J. Bichon, Quelques nouvelles déformations du groupe symétrique, C. R. Acad. Sci. Paris 330 (2000), 761-764.

[3] C. Curtis and I. Reiner, Methods of Representation Theory with applications to finite groups and orders, I, Wiley-Interscience, 1990.

[4] V. Drinfeld, Quantum groups, Proc. Int. Congr. Math., Berkeley (1987), 798-820.

[5] M. Enock and L. Vainerman, Deformation of a Kac algebra by an abelian subgroup, Commun. Math. Phys. 178 (1996), 571-596.

[6] P. Etingof and S. Gelaki, Semisimple Hopf algebras of dimension pq are trivial, J. Algebra 210 (1998), 664-669.

[7] _ The representation theory of cotriangular semisimple Hopf algebras, Int. Math. Res. Not. 1999 (1999) 387-394.

[8] _ The classification of finite dimensional triangular Hopf algebras over an algebraically closed field of char 0, Mosc. Math. J. 3 (2003), 37-43.

[9] S. Gelaki and S. Westreich, On semisimple Hopf algebras of dimension pq, Proc. Am. Math. Soc. 128(2000), 39-47; Proc. Am. Math. Soc. 128 (2000), 2829-2831.

[10] I. Isaacs, Character theory of finite groups, Academic Press, 1976.

[11] S. Montgomery, Classifying finite dimensional semisimple Hopf algebras, Contemp. Math. 229 (1998), 265-279.

[12] S. Montgomery and S. Whiterspoon, Irreducible representations of crossed products, J. Pure Appl. Algebra 129 (1998), 315-326.

[13] A. Masuoka, The $p^{n}$-th Theorem for Hopf algebras, Proc. Amer. Math. Soc. 124 (1996), 187195.

[14] _ Self dual Hopf algebras of dimension $p^{3}$ obtained by extension, J. Algebra 178 (1995), 791-806.

[15] M. Movshev, Twisting in group algebras of finite groups, Func. Anal. Appl. 27 (1994), 240-244.

[16] S. Natale, Semisolvability of semisimple Hopf algebras of low dimension, Memoirs Amer. Math. Soc. 186 (2007).

[17] W. Nichols and M. Richmond, The Grothendieck group of a Hopf algebra, J. Pure Appl. Algebra 106 (1996), 297-307.

[18] W. Nichols and M. Zoeller, A Hopf algebra freeness theorem, Am. J. Math. 111 (1989), 381-385.

[19] D. Nikshych, $K_{0}$-rings and twisting of finite dimensional semisimple Hopf algebras, Commun. Algebra 26 (1998), 321-342; Commun. Algebra 26 (1998), 2019.

[20] D. Radford, On the antipode of a quasitriangular Hopf algebra, J. Algebra 151 (1992), 1-11.

[21] _ Minimal quasitriangular Hopf algebras, J. Algebra 157 (1993), 285-315.

[22] J. Robinson, A course in the theory of groups, Springer-Verlag, 1995. 
[23] P. Schauenburg, Hopf bi-Galois extensions, Comm. Algebra 24 (1996), 3797-3825.

[24] D. Tambara and S. Yamagami, Tensor categories with fusion rules of self-duality for finite abelian groups, J. Algebra 209 (1998), 692-707.

[25] L. Vainerman, 2-cocycles and twisting of Kac algebras, Commun. Math. Phys. 191 (1998), 697-721.

[26] Y. Zhu, Hopf algebras of prime dimension, Int. Math. Res. Not. 1 (1994), 53-59.

Facultad de Matemática, Astronomía y Física, Universidad Nacional de Córdoba, Ciem - COnicET, (5000) Ciudad Universitaria, Córdoba, Argentina

E-mail address: galindo@mate.uncor.edu

Facultad de Matemática, Astronomía y Física, Universidad Nacional de Córdoba, Ciem - COnicet, (5000) Ciudad Universitaria, Córdoba, Argentina

E-mail address: natale@mate.uncor.edu

$U R L$ : http://www.mate.uncor.edu/natale 